\title{
Inspiratory muscle workload due to dynamic intrinsic PEEP in stable COPD patients: effects of two different settings of non-invasive pressure-support ventilation
}

\author{
M. Vitacca1, B. Lanini1, S. Nava2, L. Barbano1, \\ R. Porta1 ${ }^{1}$, E. Clini3 3 N. Ambrosino4
}

ABSTRACT: Inspiratory muscle workload due to dynamic intrinsic PEEP in stable COPD patients: effects of two different settings of non-invasive pressure-support ventilation. M. Vitacca, B. Lanini, S. Nava, L. Barbano, R. Porta, E. Clini, N. Ambrosino.

Background: In severe stable hypercapnic COPD patients the amount of pressure time product (PTP) spent to counterbalance their dynamic intrinsic positive end expiratory pressure (PEEPi,dyn) is high: no data are available on the best setting of non invasive pressure support ventilation (NPSV) to reduce the inspiratory muscle workload due to PEEPi,dyn.

Methods: The objectives of this randomised controlled physiological study were: 1 . To measure the inspiratory muscle workload due to PEEPi,dyn 2. To measure the effects on this parameter of two settings of NPSV in stable COPD patients with chronic hypercapnia admitted in a Pulmonary Division of two Rehabilitation Centers. Twenty-three stable COPD patients with chronic hypercapnia on domiciliary nocturnal NPSV for $30 \pm 20$ months were submitted to an evaluation of breathing pattern, PEEPi,dyn, inspiratory muscle workload and its parti- tioning during both assisted and unassisted ventilation. Two settings of NPSV were randomly applied for $30 \mathrm{~min}$ utes each: i- "at patient's comfort" (C): Inspiratory pressure support (IPS) was the maximal tolerated pressure able to reduce awake $\mathrm{PaCO}_{2}$ with the addition of a pre-set level of external PEEP (PEEPe); ii- "physiological setting" (PH): the level of IPS able to achieve a $>40 \%$ and $<90 \%$ decrease in transdiaphragmatic pressure in comparison to spontaneous breathing (SB). A PEEPe level able to reduce PEEPi,dyn by at least $50 \%$ was added.

Results: During SB the tidal diaphragmatic pressuretime product (PTPdi/b) was $17.62 \pm 7.22 \mathrm{cmH}_{2} \mathrm{O} * \mathrm{sec}$, the component due to PEEPi,dyn (PTPdiPEEPi,dyn) being $38 \pm 17 \%$ (range: $16-65 \%$ ). Compared to SB, PTPdiPEEPi,dyn was reduced significantly with both settings, the reduction being greater with $\mathrm{PH}$ compared to $\mathrm{C}$.

Conclusions: In conclusion in severe COPD patients with chronic hypercapnia the inspiratory muscle workload due to PEEPidyn is high and is reduced by NPSV at a greater extent when ventilator setting is tailored to patient's mechanics.

Monaldi Arch Chest Dis 2004; 61: 2, 81-85.

Keywords: Non-invasive mechanical ventilation, Respiratory Failure, Respiratory muscles, Hypercapnia, Breathing pattern.

${ }^{1}$ Divisione di Pneumologia Riabilitativa, Fondazione S. Maugeri IRCCS, Istituto Scientifico di Gussago, 2 Divisione di Pneumologia Riabilitativa, Fondazione S. Maugeri IRCCS, Istituto Scientifico di Pavia, ${ }^{3}$ Fondazione ONLUS Villa Pineta Gaiato (MO), 4 Cardio-Thoracic Department, University Hospital of Pisa, Italy.

Correspondence: Michele Vitacca MD; Fondazione S. Maugeri I.R.C.C.S; Istituto Scientifico di Gussago; Via Pinidolo 23; 25064 Gussago (BS) Italy; e-mail: mvitacca@fsm.it

\section{Introduction}

Non-invasive Pressure Support Ventilation (NPSV) with and without some level of Positive End-Expiratory Pressure (PEEP) is the most common mode of providing ventilatory assistance in the management of Chronic Ventilatory Failure (CVF). In stable COPD patients with chronic hypercapnia, NPSV was effective in unloading inspiratory muscles independent of whether the ventilator was set on the basis of patient's comfort or tailored to patient's respiratory muscle effort and mechanics [1].

The inspiratory effort of these patients is divided into two components. The first is represented by an isometric contraction of the inspiratory muscles to counterbalance the dynamic intrinsic positive end-expiratory pressure (PEEPi,dyn) while the second is an isotonic contraction that produces inspiratory flow. In tracheostomised ventilator-dependent patients with COPD, Appendini et al. [2] showed that the diaphragmatic pressure-time product PTP (PTPdi), an index of inspiratory muscle workload, due to PEEPi,dyn (PTPdiPEEPi,dyn) was about $40 \%$ of total PTPdi.

To the best of our knowledge, no data is available in severe stable hypercapnic COPD patients on the amount of PTP spent to counterbalance their PEEPi,dyn. Furthermore no information is available on the best setting of NPSV to reduce the inspiratory muscle workload due to PEEPi,dyn. Therefore we measured the PTPdiPEEPi,dyn in 
stable COPD patients with chronic hypercapnia. Furthermore we evaluated the effects on this parameter of two settings of NPSV: i) at patient's comfort (C) and ii) a "physiological setting" (PH) tailored to patient's pulmonary mechanics in stable COPD patients with chronic hypercapnia [1].

\section{Methods}

The investigative protocol was approved by the Institutional Ethic Committee (S. Maugeri Foundation, Italy) and was conducted according to the declaration of Helsinki. Informed consent was obtained from all the patients before enrolment into the study.

\section{Patients}

Twenty-three COPD patients with chronich ventilatory failure on long-term oxygen therapy (LTOT) and domiciliary nocturnal NPSV were studied in the Pulmonary Divisions of Gussago (9 patients) and Montescano (14 patients), S. Maugeri Foundation, as a part of a study, the results of which have been published else-where [1]. At the time when they were recruited for this study, all patients were in a stable condition, free from exacerbation for at least 1 month. Criteria for home NPSV has been detailed elsewhere [1], their demographic, anthropometric and functional characteristics are shown in table 1.

\section{Measurements}

Lung volumes and FVC were measured by means of constant volume body plethysmographs and spirometric values $\left(\mathrm{FEV}_{1}, \mathrm{FVC}\right)$ using a water spirometer. The predicted values of Quanjer [3] were used. $\mathrm{PaO}_{2}, \mathrm{PaCO}_{2}$, and $\mathrm{pH}$ were measured on blood sample from the radial artery by means of automated analyzers.

Assessment of inspiratory muscles and mechanics been detailed elsewhere [1]. Briefly, flow was measured by means of a heated pneumotachygraph connected to a differential pressure

Table 1. - Demographic, anthropometric and functional characteristics of patients in study

\begin{tabular}{lc}
\hline Age, yr & $68 \pm 5$ \\
Sex & \\
$\quad$ Female & 2 \\
$\quad$ Male & 21 \\
Weight, kg & $71 \pm 14$ \\
BMI & $25 \pm 4.6$ \\
FEV $_{1}, \%$ prd & $23 \pm 7$ \\
FVC, \% prd & $40 \pm 8$ \\
FEV & $/$ VC \% \\
RV, \% prd & $40 \pm 10$ \\
TLC, \% prd & $189 \pm 42$ \\
Home NPPV, months & $127 \pm 27$ \\
\end{tabular}

Definition of abbreviations: BMI $=$ Body Mass Index transducer and inserted between the nasal mask and the "plateau valve" of the ventilator circuit. Volume was obtained by numerical integration of the flow signal. The pressure at the airway opening (Pao) was measured at a side port placed between the mask and the pneumotachograph. Changes in pleural and abdominal pressures were estimated from changes in oesophageal (Poes) and gastric (Pga) pressures, respectively, by means of the balloon-catheter technique. Transpulmonary and transdiaphragmatic (Pdi) pressures were obtained by subtraction of Poes from Pao and Pga respectively.

\section{Data Analysis}

PEEPi,dyn and changes in the magnitude of the inspiratory effort of the diaphragm was assessed measuring Pdi swings and the tidal PTPdi (PTPdi/b) according to Appendini et al. [1, 4]. The PTPdiPEEPidyn was calculated as the difference between the area subtended from the onset of inspiratory effort to the end of inspiratory flow (total PTPdi) and the area subtended between the onset and the end of inspiratory flow (sum of resistive and elastic components) [2]. PTPdiPEEPidyn was expressed as absolute values and also as a percentage of the total PTPdi.

\section{Experimental Procedure}

The experimental setting has been detailed elsewhere [1]. NPSV was delivered by means of a portable ventilator (BIPAP, Respironics, Murrysville, PA, USA) through the commercial nose mask used at home by each patient connected to the pneumotachograph. The ventilator circuit was equipped with the Sanders NRV-2 valve (Respironics, Murrysville, PA, USA) to prevent $\mathrm{CO}_{2}$ rebreathing. All measurements were recorded as a fraction of inspired oxygen $\left(\mathrm{FI} \mathrm{O}_{2}\right)$ of 0.21 .

At the beginning of the study (baseline) and in the intervals between different settings, the subjects breathed spontaneously through the pneumotachograph for about 20-25 minutes, once removed the ventilator tubing. A set of data was collected while the patients breathing spontaneously. At the end of this baseline recording patients performed NPSV in according to two settings [1]:

i) "at patient comfort" (C): maximal tolerated IPS; the level of PEEPe was progressively increased by $1 \mathrm{cmH}_{2} \mathrm{O}$ at patient's comfort but no more than $6 \mathrm{cmH}_{2} \mathrm{O}$.

ii) "physiological setting" ( $\mathrm{PH})$ : the level of IPS able to achieve a $>40 \%$ and $<90 \%$ decrease in Pdi in comparison to spontaneous breathing (SB), avoiding a positive deflection of Pdi. A PEEPe level able to reduce PEEPi,dyn by at least $50 \%$ was added. Due to the characteristics of the portable ventilator used, a default $2 \mathrm{cmH}_{2} \mathrm{O}$ PEEPe was always added.

Modes were applied in random order, each mode of support lasting 30 to 45 minutes and the trials were separated by returning to SB for 20-25 minutes. All physiological signals were recorded 
in the last three minutes of each unassisted or assisted breathing period.

\section{Statistical analysis}

Results are expressed as a mean \pm 1 SD. Differences between treatments and within treatment were evaluated by analysis of variance (ANOVA) for repeated measures. Differences between paired groups of data were evaluated with post-Hoc paired $\mathrm{T}$ test with Bonferroni adjustment and were applied as requested by ANOVA interaction. A $p$ value $<0.05$ was considered significant.

\section{Results}

All patients tolerated NPSV well throughout the procedure. NPSV was able to significantly ( $\mathrm{p}<$ 0.01 ) improve both $\mathrm{PaO}_{2}$ (from $49.7 \pm 5.5$ to 55.1 \pm 7.7 and to $54.6 \pm 7.5 \mathrm{mmHg}$ with $\mathrm{C}$ and $\mathrm{PH}$ respectively) and $\mathrm{PaCO}_{2}$ (from $58.3 \pm 7.0$ to $53.0 \pm$ 6.1 and to $53.0 \pm 6.1 \mathrm{mmHg}$ respectively) independent of the applied setting. Arterial $\mathrm{pH}$ increased non-significantly with both settings of NPSV (from $7.39 \pm 0.02$ to $7.40 \pm 0.03$ and to 7.40 \pm 0.02 with $\mathrm{C}$ and $\mathrm{PH}$ respectively). As detailed elsewhere [1], the mean $\mathrm{C}$ setting was: IPS $=16 \pm$ $3 \mathrm{cmH}_{2} \mathrm{O}$, PEEPe $=3.6 \pm 1.4 \mathrm{cmH}_{2} \mathrm{O}$ whereas mean PH setting was: IPS $=15 \pm 3 \mathrm{cmH}_{2} \mathrm{O}$ and PEEPe $=3.1 \pm 1.6 \mathrm{cmH}_{2} \mathrm{O}$. During SB the dyn PT$\mathrm{Pdi} / \mathrm{b}$ was $17.62 \pm 7.22 \mathrm{cmH}_{2} \mathrm{O}$ sec. PTPdiPEEPidyn being $6.72 \pm 4.38 \mathrm{cmH}_{2} \mathrm{O}$ sec (fig. 1) that is 38 $\pm 17 \%$ of the total and was significantly reduced with both settings compared to SB $(\mathrm{p}<0.01)$ and it was $29 \pm 23 \%$ of the total PTP/b with $\mathrm{C}$ and $20 \pm$ $21 \%$ with PH (fig. 1); this difference was statistically significant $(\mathrm{p}<0.04)$. Fig. 2 shows from top to bottom the distribution of values for PTPdiPEEPidyn while patients breathed spontaneously, during $\mathrm{C}$ and $\mathrm{PH}$ setting respectively. The coefficiency of the variability (CV) for values of PTPdiPEEPidyn measured under $\mathrm{PH}(\mathrm{CV}=75 \%)$ was statistically high but still less $(\mathrm{p}<0.05)$ when compared to $\mathrm{C}(\mathrm{CV}=90 \%)$ (fig. 2$)$.

\section{Discussion}

This study in stable severe COPD patients with chronic hypercapnia demonstrated that the inspiratory muscle workload due to PEEPidyn is high and is reduced by NPSV at a greater extent when PEEPe is tailored to patient's mechanics.

To the best of our knowledge this is the first report of PTPdiPEEPi,dyn in these stable patients. In acutely ill, ventilated COPD patients [2] it has been shown that the PTPdiPEEPi,dyn is about $40 \%$ of the total PTP. Stable COPD patients with CVF of our study show similar proportion of PTPdiPEEPi,dyn related to total PTP indicating that also in these stable patients, PEEPdyn may represent a relevant burden to be taken into account when deciding NPPV.

In this study we used PTPdi as an index of inspiratory muscle workload. PTPdi, is closely related to oxygen cost of breathing and has several advantages over measurements of mechanical work of breathing [5].

It has been shown [6] that in stable COPD patients the addition of $5 \mathrm{cmH}_{2} \mathrm{O}$ PEEPe to PSV 10 $\mathrm{cmH}_{2} \mathrm{O}$ resulted in further $42 \%$ reduction of PTPdi, a value very similar to that of baseline PTPdiPEEPi,dyn of our study confirming that addition of PEEPe is able to reduce the inspiratory workload due to PEEPi,dyn. In our study NPSV tailored to the

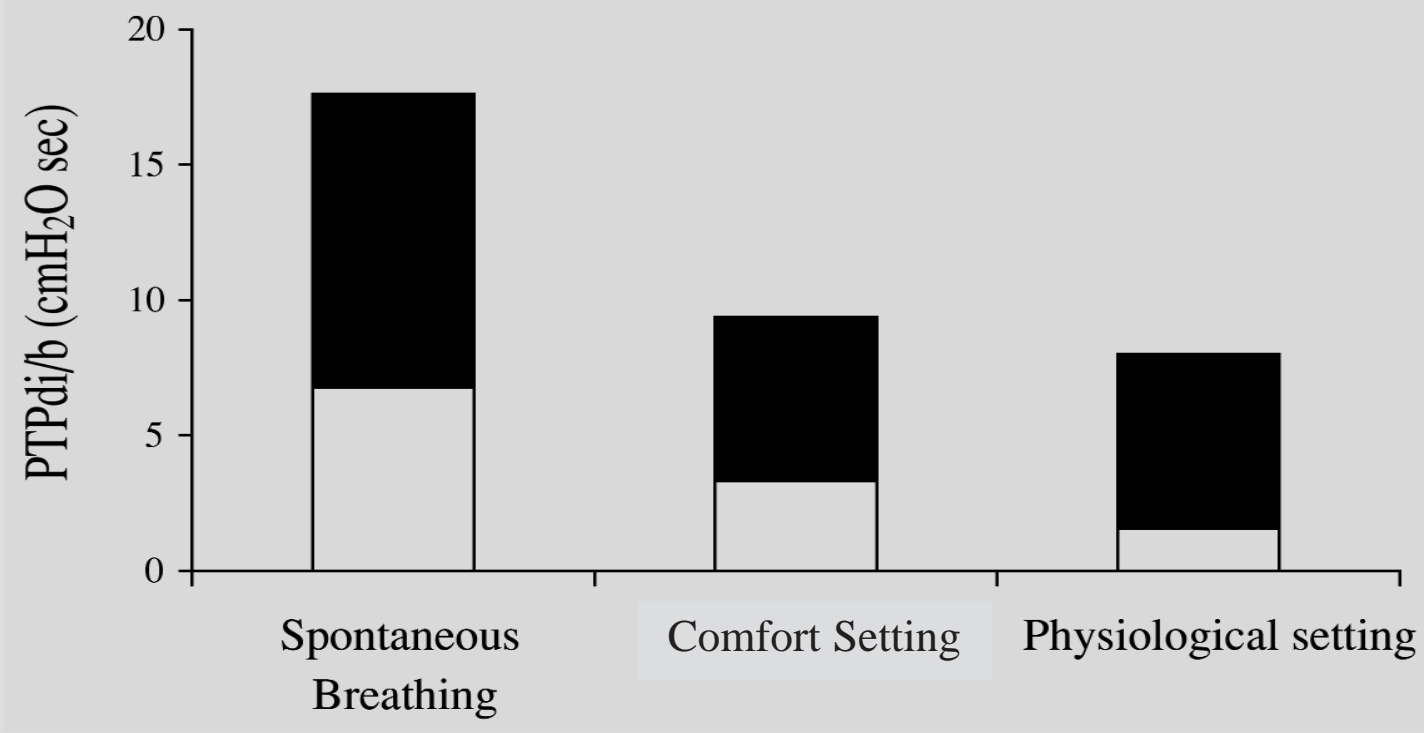

Fig. 1. - Baseline (SB) and changes under comfort setting (C) and physiological setting (PH) of mean values of the total PTPdi/b. The different bars indicate PTPdi-PEEPidyn (white bar) and the sum of resistive and elastic components (dark bar) of the PTPdi per breath (PTPdi/b). $\$$ p $<0.01$ vs $\mathrm{SB} ; * \mathrm{p}<0.04$ vs ES. 


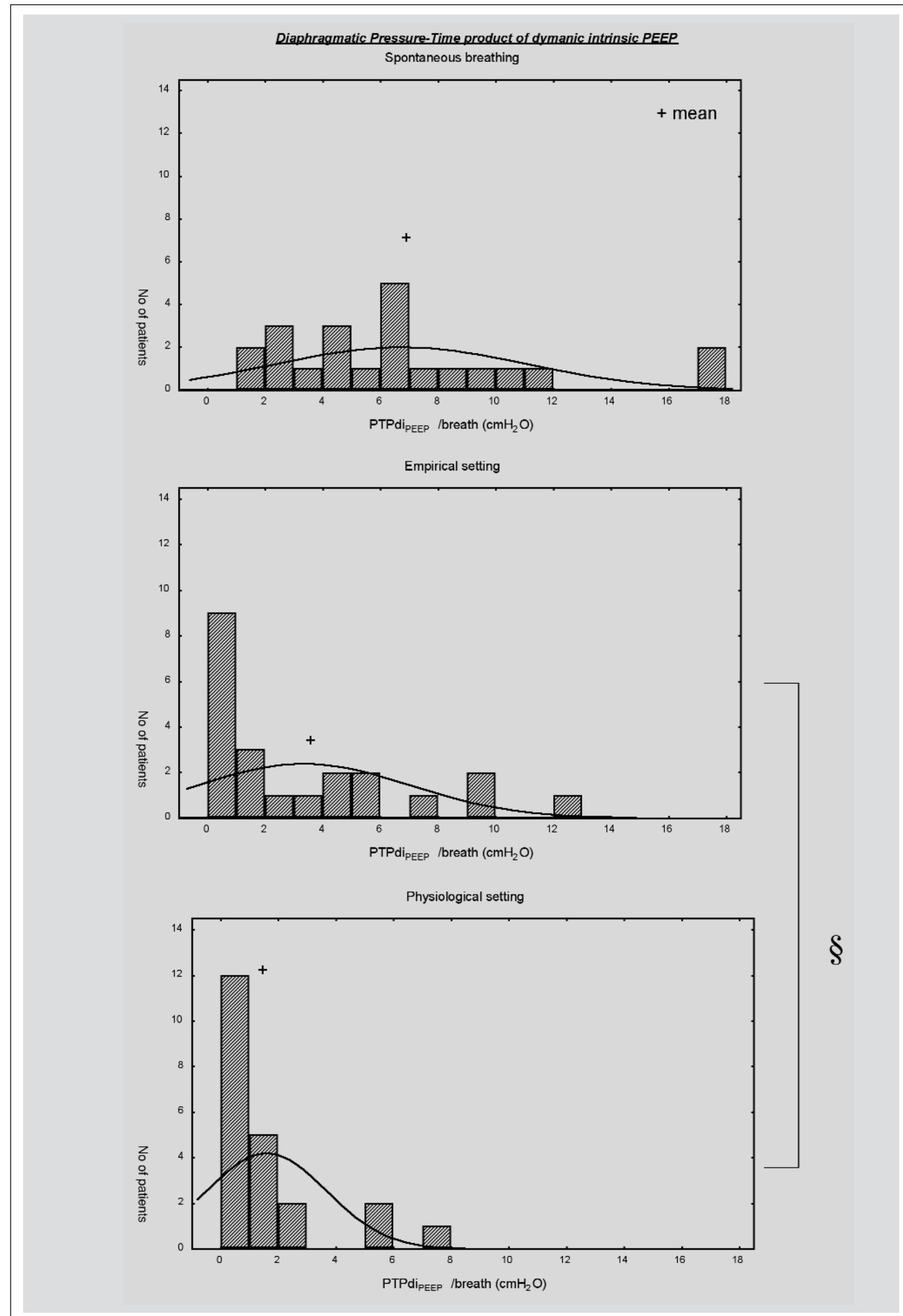

Fig. 2. - From top to bottom the distribution of values for PTPdi-PEEPidyn while patients breathed spontaneously (SB), during C and PH setting respectively. For comment see text. $\S p<0.05$. 
patient's respiratory muscle effort and mechanics was able to reduce PTPdiPEEPi,dyn more effectively than when set at comfort (figure 1 and 2).

Whether this finding implies the need of routinely set NPSV in these stable patients by invasive assessment of inspiratory muscles and lung mechanics is still debatable.

\section{References}

1. Vitacca M, Nava S, Confalonieri M, Bianchi L, Porta R, Clini E, Ambrosino N. The appropriate setting of noninvasive Pressure Support Ventilation in stable COPD patients. Chest 2000; 118; 1286-1293.

2. Appendini L, Purro A, Ptessio A, Zanaboni S, Carone M, Spada E, Donner CF, Rossi A. Partitioning of inspiratory muscle workload and pressure assistence in ven- tilator-dependent COPD patients. Am J Respir Crit Care Med 1996; 154: 1301-1309.

3. Quanjer PH. Working Party on "Standardization of lung function tests". Bull Eur Physiopathol Respir 1983; 19 (Suppl 5) 7-10.

4. Appendini L, Patessio A, Zanaboni S, Carone M, Gukov B., Donner CF, Rossi A. Physiological effects of positive end expiratory pressure and mask pressure support during exacerbations of chronic obstructive pulmonary disease. Am J Respir Crit Care Med 1994; 149: 1069-1076.

5. Tobin MJ. Monitoring respiratory mechanics in spontaneously breathing patients. In Tobin MJ. Editor Principles and practice of intensive care monitoring. New York: Mc Graw-Hill 1998; 617-653.

6. Nava S, Ambrosino N, Rubini F, Fracchia C, Rampulla C, Torri G, Calderini E. Effect of nasal pressure support ventilation and external PEEP on diaphragmatic activity in patients with severe stable COPD. Chest 1993; 103: 143-150.

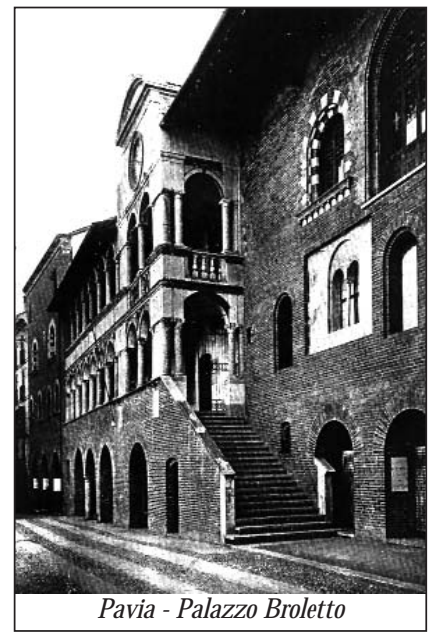

